\title{
Desarrollo de un dispositivo de observación de clases de Educación Física para la enseñanza básica: una perspectiva naturalista desde la experiencia docente
}

\author{
Sergio Toro-Arevalo* \\ Ivan Oliva** \\ John Quintero Tapia***
}

\begin{abstract}
Resumen: El presente trabajo sistematiza el abordaje cualitativo de un proyecto patrocinado por el Ministerio de Educación de Chile, en función de desarrollar un instrumento de observación de las clases de educación Física para el Nivel de Enseñanza General Básica en establecimientos con financiamiento público. El mencionado instrumento tiene como objetivo favorecer procesos de diálogo disciplinar y fortalecer el desempeño didáctico de las/os profesionales del área. El trabajo cualitativo se desarrolló en etapas sucesivas y complementarias: revisión de literatura específica (fase teórica), percepciones de estudiantes de último año de la disciplina (fase ingenua) y de profesionales en ejercicio laboral (fase naturalista). De la confluencia de dichas fuentes, se establecieron tres dimensiones del desempeño didáctico, a saber: gestión, acción y saber didáctico. Como resultado se logró saturar, depurar y definir los indicadores de cada dimensión, como también generar y triangular indicaciones de implementación para el desarrollo de un dispositivo de observación de clases de Educación Física para la Enseñanza Básica en Chile. El presente reporte de investigación discute principalmente los resultados y protocolos metodológicos asociados a la fase naturalista.
\end{abstract}

Palabras clave: Educación Primaria y Secundaria. Educación Física. Observación. Análisis cualitativo.

\footnotetext{
"Instituto de Filosofía y Estudios Educacionales. Campus Isla Teja. Universidad Austral de Chile, Valdivia. Chile. E-mail: seatoro@gmail.com

"Instituto de Filosofía y Estudios Educacionales. Campus Isla Teja. Universidad Austral de Chile, Valdivia. Chile. E-mail: olivauach@gmail.com

"'Pedagogía en Educación Física. Universidad San Sebastián, sede Valdivia. Chile. Email: johnjamer@gmail.com<mailto:johnjamer@gmail.com>
} 


\section{ANIECEDENTES GENERALES}

El mejoramiento de la enseñanza y de los resultados de aprendizaje en el sector de Educación Física constituye un objetivo central para el Ministerio de Educación de Chile (MINEDUC). En este contexto, el aseguramiento de la calidad en los procesos de gestión pedagógica invita al desarrollo e implementación de nuevos dispositivos, que situados en el propio establecimiento, desafíen al perfeccionamiento permanente de sus profesores ${ }^{1}$.

En este contexto, se propuso la caracterización de las distintas formas que se adoptan para la enseñanza de la Educación Física en Educación Básica, en los establecimientos subvencionados del país, respondiendo a la siguiente pregunta orientadora ¿Cúales son las dimensiones a considerar en la elabroación de un intrumento de observación de clases de educación fisica de excelencia, desde la perspectiva de las practicas consideradas exitosas y de actores en ejercicio? En esta dirección, y en atención a los términos de referencia planteados por el Minesterio de Educación de Chile, se configuraron los objetivos que se presentan a continuación: i) Elaborar un dispositivo de observación de las clases de Educación Física; ii) Proponer una categorización de las tareas motrices según niveles de complejidad; iii) Dar validez y confiabilidad a los instrumentos y métodos de análisis de los datos recogidos.

La investigación contempló tres niveles metodológicos: teórico, ingenuo y naturalista (Figura 1) orientados a la sistematización y confluencia de los diferentes tipos de información obtenida, y que en definitiva, permitieron la construcción de un instrumento de observación de las clases de Educación Física en la Educación General Básica (EGB). La conjunción de estos tres niveles metodológicos tuvo como objetivo la especificación y pertinencia de las categorías

\footnotetext{
${ }^{1}$ En este contexto se firmó el convenio denominado: Convenio entre el Ministerio de Educación de la República de Chile y la Universidad Austral de Chile sobre el Servicio de Elaboración de Instrumentos de Observación de Clases de Educación Física en la Enseñanza Básica" № 9646 y cuya Resolución Exenta es 9735 del 30 de diciembre del 2009
} 
a observar y su acoplamiento a las dimensiones establecidas como niveles globales del desempeño del docente, a saber: gestión didáctica, acción didáctica y saber didáctico.

Cada uno de las dimensiones mencionadas, las cuales en un primer momento emanaron de la revisión bibliográfica e investigativa, pretenden visualizar el desempeño del docente de acuerdo a aspectos que se configuran en virtud del aprendizaje de los y las escolares en terminos de las acciones emprendidas y que son identificables y reconocidas por pares docentes. En este sentido, las diferentes etapas de la investigación permitieron ir estructurando y refinando tanto los límites y fronteras de cada dimensión, en la medida que se depuraban los procesos de análisis y la explicitación de los indicadores de cada una de ellas. En este sentido la observación y explicitación de los criterios de observación de los docentes, tanto ingenuos como naturales, se convitió en el nucleo eje para la operacionalización de las dimensiones en indicadores que permitieran el dialogo y explicitación, tanto de los supuestos didácticos y la base disciplinaria con la cual los docentes se desenvuelven en sus respectivas clases o sesiones.



Figura 1. Diseño metodológico global.

Fuente: De los autores

Movimento, Porto Alegre, v. 19, n. 01, p. 161-181, jan/mar de 2013. 
En este contexto se llevó a cabo un proceso de investigación que permitiera dar cuenta de los objetivos señalados anteriormente Esta situación es de relevancia sustantiva en el contexto nacional, dada el numero de intituciones formadoras de profesionales de la Educación Física y la escasa información disponible acerca de la formación y operacionalización de procesos didácticos en el escenario de desempeño profesional, tanto a nivel de convergencias, como de divergencias epistemológicas-paradigmáticas y sus efectos en aprendizaje escolar. Lo anterior, atendiendo a la especificidad del nivel de enseñanza general básica, como en la profundidad y desarrollo de los diversas estretegías didácticas y de contenido implementadas.

En base a lo anterior, se exponen los principios globales metodológicos, para posteriormente profundizar en el nivel metodológico naturalista, dando cuenta de la consitencia y coherencia del modelo implementado. Los principales antecedentes de cada nivel o fase se exponen en forma sintetica en el esquema 1, permitiendo una visión general del trabajo, sus etapas y sus niveles de articualción.

Cada una de estas etapas se describe de la suguiente forma:

a) Fase construcción teórica: La fase de construcción teórica consideró una revisión sistemática de la literatura nacional e internacional sobre el tema, dando cuenta de la diversidad de antecedentes conceptuales y metodológicos asociados a las categorías de observación e interpretación de los procesos de enseñanza-aprendizajes del subsector de Educación Física. Cabe señalar que este nivel fue transversal en todo el proceso del estudio. Se revisaron diferentes corrientes referidas a la clase o lección de la disciplina, Ahumada (2005) Blázquez (2000), Castañer y Camerino (1996), Ramos y Del Villar (2005) Lopez Pastor (1999, 2006), Pastor Pradillo (2007), Sanmartí (2007), Topsch (2004), caracterizando los elementos o categorías que describen un pertinente desarrollo de los diferentes procesos, tanto generales como particulares. Paralelamente se estructuró una revisión de corrientes actuales sobre el fenómeno del aprendizaje y cognición, a fin de establecer un derrotero epistémico de mayor complejidad en relación a las dinámicas 
observado-observador y la construcción de conocimiento. En tal sentido se optó por autores posicionados desde una perspectiva de la cognición como proceso y fenómeno encarnado y situado (GALLAGHER, 2010-2008; NÖ̈, 2010; COSMELLI E IBAÑEZ, 2007; FREEMAN, 2007; THOMPSON, 2007; VARELA, 2000; HUBRIG; HERRMANN, 2010), considerando que dichas perspectivas confluyen con los propósitos metodológicos y epistemológicos del dispositivo de observación de clases de Educación Física desarrollado.

b) Fase de revisión ingenua: El objetivo de esta fase fue evaluar una pauta de observación preliminar (construida desde la fase teórica), a partir de una mirada no especializada pero con grados significativos de experiencia y agenciamiento con el fenómeno. Para esta etapa se consideró la visualización de clases filmadas de Educación Física, previamente seleccionadas por dominios de aprendizajes. En base a esta selección de videos, se realizó una aplicación piloto de la pauta por parte de 56 estudiantes $^{2}$ de la disciplina próximos a finalizar el programa de formación, los cuales evaluaron la ausencia y presencia de los descriptores asociados a los dominios de acción, gestión y saber didáctico. La ejecución de esta fase permitió calibrar el instrumento y comenzar la discusión respecto de su estructura, organización, extensión y contenido.

c) Fase naturalista: El proceso de mejoramiento de la pauta contempló la evaluación cualitativa de profesores del sistema escolar. Se extrajo de esta discusión, insumos para precisar los descriptores con un leguaje más pertinente y contextualizado a los profesores de aula. Luego de esta discusión, los profesores observaban la pauta y entregaban aportes para su mejoramiento.

\footnotetext{
${ }^{2}$ Estudiantes de tercer y cuarto año de la carrera de Pedagogía en Educación Física, Deportes y Recreación de la Universidad Austral de Chile.
} 
A partir del programa global de investigación, el presente artículo expone las directrices metodológicas y los resultados del trabajo de campo asociado fundamentalmente a la fase naturalista descrita precedentemente.

\section{Metodología}

Se realizó una fase metodológica naturalista orientada a rescatar los esquemas de observación y aportes conceptuales de profesores(as) de Educación Física que se desempeñan en el sistema educativo público-subvencionado chileno. Esta fase respondió a la necesidad de generar información cualitativa, validada y triangulada, susceptible de ser integrada a las bases conceptuales que sustentan el desarrollo de un dispositivo de observación de las clases de Educación Física. Desde esta base, se configuró el siguiente diseño metodológico.

\subsection{TÉCNICAS DE RECOLECCIÓN DE INFORMACIÓN}

Se implementaron grupos focales (eventos comunicativos dirigidos pero no controlados), dada su densidad para la construcción multicriterial y por sus potenciales participativas y de autoconocimiento grupal, permitiendo convertir los colectivos en dispositivos dinámicos de autorreflexión (ESPINA, 2007). Con ello, se estableció un doble parámetro de triangulación: i) inter-técnicas, a través de Grupos de Discusión en grado secuencial complejo; ii) teoría sustantiva integrada a teoría formal (GLASSER; STRAUSS, 1967). Ambas instancias representadas respectivamente por la información emergente-organizada de los sujetos y por la matriz de observación establecida en la fase teórica de la investigación.

Se planificó el desarrollo de dos grupos focales en la siguiente secuencia: el primero de tipo exploratorio (apertura) y el segundo de tipo confirmatorio (focalización). El primero, generó información no contemplada en la pauta de observación preliminar estructurada 
deductivamente, a la vez que confirmó la coherencia con categorías ya establecidas. El segundo, obedeció a profundizar y confirmar las categorías emergentes en el grupo focal primario, y que no fueron contempladas en la pauta preliminar. Cabe mencionar que en los dos grupos focales participaron 10 y 11 docentes respectivamente.

Con ello se integra, en la perspectiva de Arnold (2004), una estrategia no sólo orientada a rescatar las formas de conocimiento individuales de cada informante, sino además, como usuarios de los medios observacionales e interpretativos disponibles en el repertorio de sus contextos de ejercicio. Con ello se configura una observación de segundo orden orientada a la observación de sistemas de observadores y sus respectivas observaciones, expandiendo el estudio y su aplicación hacia la identificación de conjuntos relacionados de distinciones y no sólo a la reducción analítica y causal de componentes y procesos aislados, en otras palabras, a la observación de procesos dinámicos en mutua afectación, es decir, redes de retroalimentaciones de observaciones que se sostienen unas a otras.

\subsection{CRITERIOS DE SELECCIÓN DE INFORMANTES CLAVES}

Dado el carácter naturalista de esta fase, se consideró la incorporación de profesores de Educación Física, en función de la representatividad social atingente al objeto de estudio. Se consideraron los siguientes criterios de selección: i) Poseer una experiencia docente de 5 a 15 años en el área de la Educación Física fundamentalmente en básica, segundo ciclo ${ }^{3}$; ii) Poseer título de profesor con o sin mención en el área; iii) No haber sido sometidos al $\mathrm{AEP}^{4}$; iv) Representatividad de Género

\footnotetext{
${ }^{3}$ En Chile, el segundo ciclo de Educación General Básica está compuesto de $4^{\circ}$ a $8^{\circ}$ año de Escolarización.

${ }^{4}$ Asignación de Excelencia Pedagógica-AEP, es un programa del Ministerio de Educación de Chile el cual procura fortalecer la calidad de la educación mediante el reconocimiento a la labor docente en términos de conocimientos, habilidades y competencias de excelencia.
}

Vovimento, Porto Alegre, v. 19, n. 01, p. 161-181, jan/mar de 2013. 


\subsection{CotAS COMUNICATIVAS DE LA FASE}

Las cotas comunicativas del estudio quedaron definidas como los espacios de comunicación pertinentes al estudio, en este caso, todos aquellos tópicos que tengan significado para los informantes en el contexto de la observación de diferentes secuencias didácticas filmadas en clases de Educación Física. El énfasis, por tanto, no estuvo en la valoración referencial de dichas secuencias por parte de los informantes para los casos específicos, sino en los campos observacionales desde los cuales fueron evaluadas e interpretadas.

\subsection{ESTRATEGIAS DE ANÁLISIS E INTERPRETACIÓN DE INFORMACIÓN}

Los grupos de discusión fueron transcritos integralmente, según el consentimiento informado por parte de los informantes, resguardando la identidad y las respectivas observaciones de dichos sujetos. Preliminarmente y mediante el paquete informático QDA Miner 3.2.2. - WordStat 6.0.1., se desarrolló un análisis de contenido preliminar orientado a validar el cumplimiento de las cotas comunicativas de la fase.

Posteriormente se procedió a un proceso de codificación que complementó estrategias de carácter inductivo y deductivo. Para ello se procedió a levantar una matriz de códigos preliminares proyectada desde la revisión de estado del arte, la cual fue aplicada al análisis y revisión ingenua de estudiantes de educación físca de último año. Atendiendo a códigos emergentes, se optó por una estrategia inductiva complementaria, la que consistió en un análisis naturalista de profesoras/es de Educación Física sobre registros audiovisuales provenientes de profesores de la misma disciplina acreedores de la Acreditación de Excelencia Pedagógica (AEP), con los cuales se desarrollaron grupos de discusión en dos ocasiones, a fin de resguardar la consistencia de las categorías emergentes. 
Desde la convergencia de estas instancias, se completó un proceso de codificación definitiva que posibilitó dos niveles de análisis. En un primer momento, se analizó y comparó la distribución de frecuencias, con el objetivo de caracterizar preliminarmente el campo observacional en el cual se movieron las distinciones del colectivo de discusión. En un segundo momento, se analizaron los efectos de asociación de los códigos desde una perspectiva sistémica. El criterio utilizado como indicador para la asociación, fue la co-ocurrencia de códigos dentro de un mismo campo semántico ${ }^{5}$. El coeficiente de Jaccard (tolerancia 0,00001) fue el indicador específico de ocurrencia de segmentos superpuestos, el cual fue la base para la construcción de dendrogramas y redes de interacción globales de códigos.

Derivado de lo anterior, fue posible complementar en conjunto con la revisión del estado del arte y la fase de observación ingenua, la focalización y congruencia general de la matriz de observación preliminar, en términos de su organización e inclusión de dimensiones de observación.

\section{Resultados}

\subsection{ANÁLISIS DE CONTENIDO PRELIMINAR}

El análisis de contenido preliminar (Figura 2) evidencia que las cotas comunicativas de la fase, en relación a las observaciones de secuencias didácticas específicas fueron respetadas. Los conceptos con mayor frecuencia están en directa relación a la clase y su interpretación.

${ }^{5}$ Para efectos de la investigación, se definió como unidad o campo semántico, a cada uno de los turnos de intervención efectuados por los informantes en relación a las preguntas de base del grupo focal. 


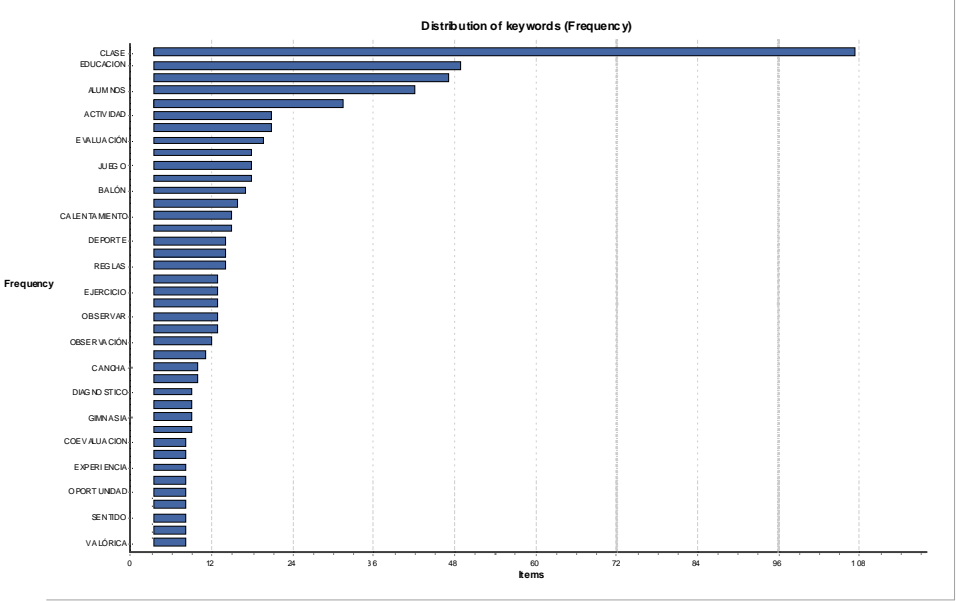

Figura 2- Análisis de contenido preliminar en base a los dos grupos de discusión desarrollados,

Fuente: De los autores

\subsection{FRECUENCIA DE CÓDIGOS}

La Figura 3 muestra la organización de la matriz categorías y los códigos asociados. En ella se muestra la frecuencia y porcentaje de códigos por categorías aplicados.

\begin{tabular}{|c|c|c|c|c|c|c|c|c|}
\hline Calegoria & Cödigo & Descripciòn & Cuenta & 4 codigos & Casos & SCASOS & 2de Palabr: & 4patabras \\
\hline Dessple gue Dodscictico & 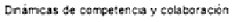 & & $T$ & $2.8 \%$ & 2 & $100,0 \%$ & 612 & $3.5 \%$ \\
\hline Despiegue Oidaction & Empatia & & 14 & $5.5 \%$ & 2 & $100,0 \%$ & 855 & $4.9 \%$ \\
\hline Desple que Didactico & Formas de Eualuacian & & 16 & $6.3 \%$ & 2 & $100,0 \%$ & 1239 & $7.3 \%$ \\
\hline Emergentes & Anticulacion Teria-Fraxs & & 26 & $10.2 \%$ & 2 & $100,0 \%$ & 2078 & $120 \%$ \\
\hline Enrergentes & Autou Cukida e Higene & & 6 & $2.4 \%$ & 2 & $100,0 \%$ & $\leq 29$ & $3,1 \%$ \\
\hline Emergentes & Conscencis Ecologsas & & 2 & $0.8 \%$ & 2 & $100.0 \%$ & 247 & $1.4 \%$ \\
\hline Energentes & Desarrobo cumunicativa y socal & & 8 & $3,1 \%$ & 2 & $100,0 \% \%$ & 1000 & $5,8 \%$ \\
\hline Emergentes & Dessmrobio de valores & & 17 & $6.7 \%$ & 2 & $100,0 \%$ & 1462 & $8.4 \%$ \\
\hline Energentes & Forrnakdad dockate & & 6 & $2.4 \%$ & 2 & $100,60 \%$ & 823 & $3,6 \%$ \\
\hline Emergentes & Lenguaje Técnico-Feda gógica & & 94 & $5.5 \%$ & 2 & $100,0 \%$ & 1044 & $6.0 \%$ \\
\hline Emerpentes & Optrazación del terron & & 13 & $5,1 \%$ & 2 & $100,0 \%$ & 1142 & $6.6 \%$ \\
\hline Emergentes & Orden y dacolins & & $a$ & $3.1 \%$ & 2 & $100,0 \%$ & 679 & $3.5 \%$ \\
\hline Emer gentes & Porticpacion y Ludarno & & 10 & $3.9 \%$ & 2 & $100,0 \%$ & 579 & $5.7 \%$ \\
\hline Emergentes & Progresion comoles de contenidos & & 98 & $3.1 \%$ & 2 & $100,0 \%$ & 1317 & $7.8 \%$ \\
\hline Erive dentilts & Mañejo de quapó & & 4 & $1.6 \%$ & 1 & $540 \%$ & 324 & $1.9 \%$ \\
\hline Emergentes & Concrentizacion de sprendizajes & & 4 & $16 \%$ & 1 & $50.0 \%$ & 247 & $1,4 \%$ \\
\hline Gestón Déactica & Herel de especificisad & & 18 & $7.1 \%$ & 2 & $100.0 \%$ & 1274 & $7.4 \%$ \\
\hline Gestión Déactics & Organcacion de ta interaccoen & & 16 & $6.3 \%$ & 2 & $100,0 \%$ & 1550 & $8.9 \%$ \\
\hline Geston Déactica & Plsnificición y etabuss de la clase & & 93 & $5.1 \%$ & 2 & $100.0 \%$ & 1104 & $6.4 \%$ \\
\hline Saber deacteon & Asocietrudad y Panticpacion & & 14 & $5.5 \%$ & 2 & $100,0 \%$ & 1340 & $7,7 \%$ \\
\hline Saber didactoo & Auto noma del estudiante & & 9 & $35 \%$ & 2 & $100.0 \%$ & 700 & $4.0 \%$ \\
\hline Saber destuxo & Impleanicat & & 11 & $4.3 \%$ & 2 & $100,0 \%$ & 642 & $3.7 \%$ \\
\hline Saber dosctes & Wheles de complejods & & & & & & & \\
\hline
\end{tabular}

Figura 3. Síntesis de frecuencias de codificación que integra la fase exploratoria y focalizada.

Fuente: De los autores

Movimento, Porto Alegre, v. 19, n. 01, p. 161-181, jan/mar de 2013. 
Los códigos asociados a la categoría emergentes fueron fundamentales para los análisis e interpretaciones posteriores, en términos de evaluar su presencia en la matriz de observación preliminar diseñada por el equipo de investigación.

Se observa en el Figura 2 una alta valoración de los códigos asociados a las categorías Gestión Didáctica, Despliegue Didáctico y Saber Didáctico, contempladas en la matriz preliminar de observación de clases. No obstante, se observa una presencia significativa de códigos emergentes, cuya pertinencia en la matriz preliminar fue evaluada desde un análisis de orden sistémico, orientado a la coherencia general del instrumento.

La emergencia de códigos como: articulación de teoría - praxis, niveles de especificidad y progresión compleja de contenidos, tiene que ver con la necesidad de optimizar el tiempo de la clase y de profundizar en cada uno de los contenidos. A modo de ejemplo, se presentan desde las tres categorías, la definición de cada uno de los códigos, así como los relatos que se asocian.

\begin{tabular}{|l|l|}
\hline \multicolumn{1}{|c|}{ Código } & \multicolumn{1}{c|}{ Relato } \\
\hline $\begin{array}{l}\text { Articulación teoría- } \\
\text { práxis }\end{array}$ & $\begin{array}{l}\text { "(la clase)...me causaba como si era teórica o práctica, o cuantas veces a la } \\
\text { semana tienen, porque yo no sé cuántas veces a la semana tiene clases él. } \\
\text { Encontré que utilizó mu cho tiempo en explicar en dar cosas teóricas y muy poco } \\
\text { tiempo en lo práctico". }\end{array}$ \\
\hline $\begin{array}{l}\text { Niveles de } \\
\text { especificidad }\end{array}$ & $\begin{array}{l}\text { "el objetivo es muy amplio... para una clase de } 40 \text { minutos el objetivo tiene que } \\
\text { ser súper centrado, o sea vamos a trabajar pase de pecho, por ejemplo y toda mi } \\
\text { clase y todas las actividades que yo realizo en función a llevarlo al final, para una } \\
\text { evaluación final también de pase de pecho"... }\end{array}$ \\
\hline $\begin{array}{l}\text { Progresión } \\
\text { compleja de } \\
\text { contenidos }\end{array}$ & $\begin{array}{l}\text { "a ver, hay otra cosa que es importante que se debe evaluar en la clase de } \\
\text { Educación Física y que uno debe considerarlo, por ejemplo siempre se debe } \\
\text { mostrar lo que se va a hacer, lento primero, lento, o sea cuando por ejemplo } \\
\text { cuando él los enfrento y les dijo entreguen el balón, entonces tiene que mostrarle } \\
\text { esto, entrego el balón, corro detrás de mí compañero, me ubico atrás, entrego el } \\
\text { balón, corro, muestro dos o tres veces lento y una vez que muestro lento y todos } \\
\text { comprendieron, hace el ejercicio rápido, pero el decía, pasen por detrás y ya al tiro } \\
\text { en 6 tiempos, 1, 2,3,4,5, 6" }\end{array}$ \\
\hline
\end{tabular}

Figura 4. Códigos y relatos

Fuente: De los autores 
Un segundo punto a considerar, tiene que ver con el Saber y Despliegue Didáctico. En esta dimensión, desde el cruce teórico y sustantivo así como la revisión del estado del arte y la observación ingenua, se coincide en la relevancia de las formas de evaluación. En específico, se plantea la importancia de las formas en como el docente evalúa permanentemente durante la clase de Educación Física.

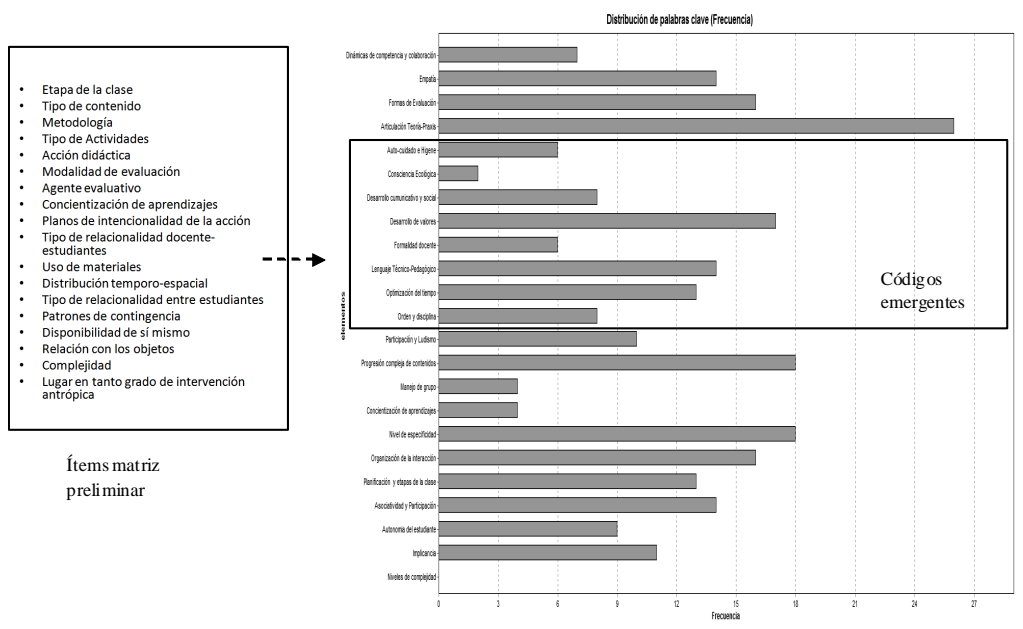

Figura 5 - Distribución de códigos asociados a la categoría emergentes.

Fuente: de los autores

Como sub-categorías emergentes, aparece la Educación Física como un espacio para el desarrollo de valores, dado que permite la asociatividad y participación de los estudiantes. Junto con ello, se observa que esto está mediado por la empatía con el docente, dado su grado de implicancia técnica y afectiva con los estudiantes. Por lo anterior, se torna importante tener en cuenta estas categorías al momento de la interpretación de la clase y el diseño del instrumento de observación. 


\subsection{ANÁLISIS DE INTERACTIVIDAD DE CÓDIGOS}

Tal como fue señalado, el indicador principal para modelar la interactividad de los diversos códigos y su importancia sistémica, fue la co-ocurrencia de códigos por unidades de sentido. En la Figura 6 una matriz muestra el patrón de co-ocurrencia de códigos de ambas instancias integradas (exploratoria y focalizada).

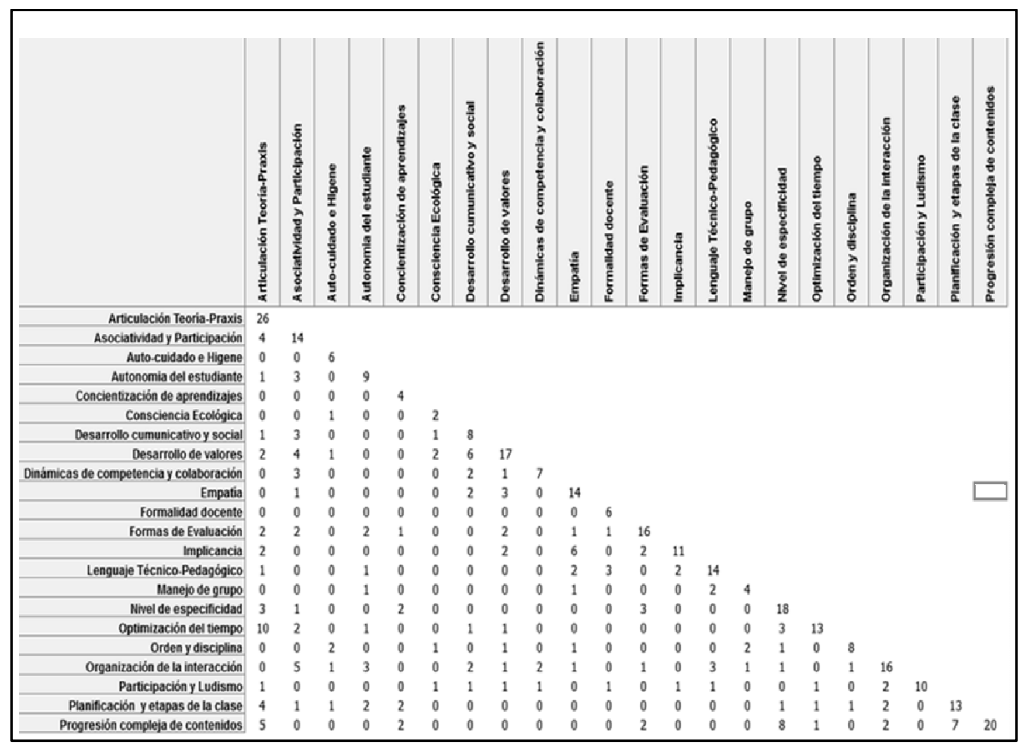

Figura 6. Matriz de co-ocurrencia de códigos integrados.

Fuente: De los autores

Desde la matriz de co-ocurrencia, se proyecta el dendrograma ${ }^{6}$ (Figura 7) como recurso de observación de los patrones de interactividad de códigos.

${ }^{6}$ Gráfica que permite evaluar las relaciones de agrupación entre los códigos desde la base del cálculo de sucesivas subdivisiones, infiriendo criterios de asociación de los mismos. 


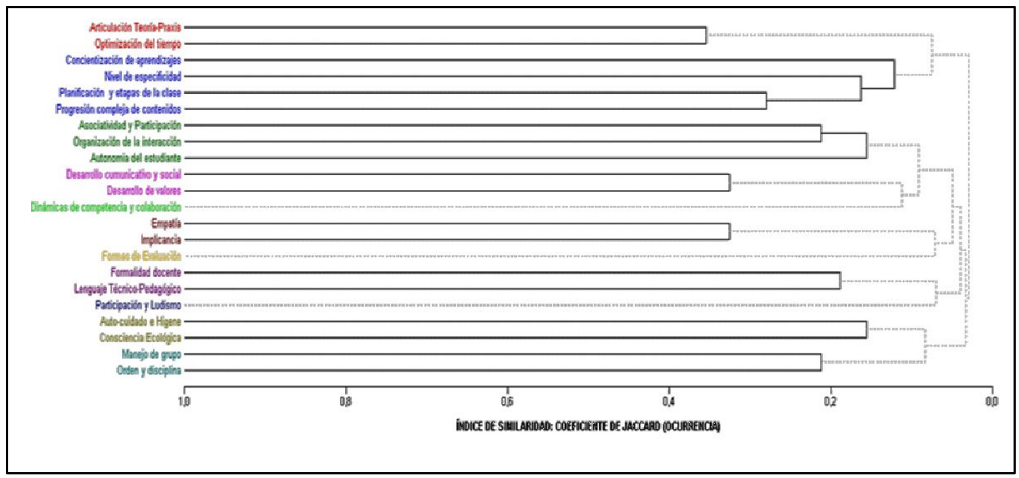

Figura 7 - Dendrograma y clústeres de códigos proyectado desde las instancias de exploración y focalización de la etapa naturalista.

Fuente: De los autores

La Figura 7 muestra en términos generales, la tendencia al agrupamiento de códigos desde la base del índice de similaridad proyectado por el coeficiente de Jaccard $^{7}$. En necesario destacar que el patrón de asociatividad graficado, emergente desde los procesos de configuración observacional del grupo focal, prescinde de la adscripción preliminar de los códigos a categorías específicas. Esto es fundamental para evaluar la coherencia general del instrumento, en función de la equivalencia de las distintas categorías y la matriz de observación previamente definida.

Este mismo patrón de co-ocurrencia se representa en la red de interactividad global de códigos (Figura 8). Desde la integración de las instancias de exploración y focalización, es posible describir en términos generales la ocurrencia de cuatro clústeres (en colores distintos), los que permiten evaluar no solo la importancia en términos de frecuencia de códigos específicos, sino además su mediación sistémica en la red global. Esto último, con el objetivo de evaluar no solo la presencia de códigos en el dispositivo de observación, sino además discutir la organización de los distintos ítems y dominios propuestos inicialmente.

${ }^{7}$ Medida de similitud referida en este caso especifico, a la co-ocurrencia de códigos dentro del mismo campo semántico. Su valores están en el rango de 0 (ausencia de similitud) a 1 (correspondencia absoluta). 


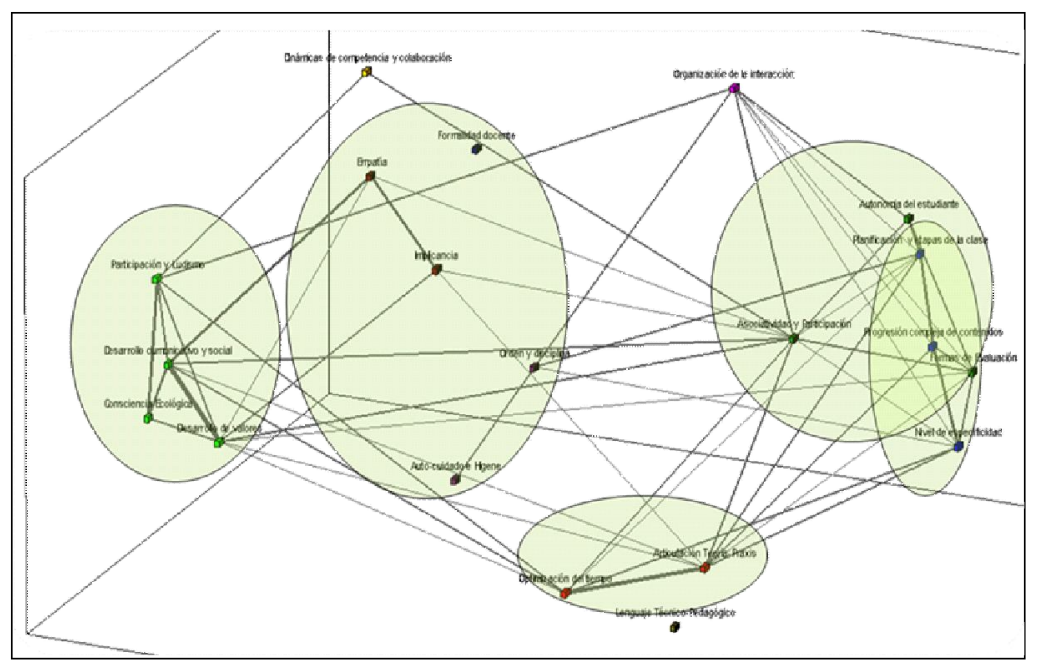

Figura 8 - Red de interactividad global de códigos y clústeres.

Fuente: De los autores

\section{Discusión FINAL}

Desde la información cualitativa levantada y triangulada desde la fase naturalista, fue posible concluir la congruencia general del instrumento con la red de interactividad de códigos y los principales clústeres observados. No obstante, es necesario rescatar los siguientes aspectos en la elaboración de un dispositivo de observación definitivo que integre los elementos observacionales y contribuciones conceptuales de profesores en ejercicio:

I. La importancia sumativa (frecuencia) y relacional (interactividad) de los códigos optimización del tiempo y articulación teoría-praxis. En otras palabras, la necesidad no solo de integrar ambos códigos, sino además asociarlos en un ítem coherente dentro del instrumento de observación definitivo. 
II. La necesidad de incluir en el dispositivo de observación de clases de Educación Física, aspectos relacionados al fomento de valores, desarrollo comunicativo-social, implicancia y empatía docente-estudiantes.

III. Reconocer la organización de la interacción como un código mediador de gran parte de los planos de reformulación de los docentes participes de los grupos focales. Esto, en términos de reconocer la importancia fundamental de los esquemas de relacionalidad entre docente y estudiantes, y su carácter transversal a todos los demás dominios de observación.

IV. Establecer que la coherencia del instrumento está sostenida en los acuerdos que se generaron en el diálogo entre profesoras/es en desempeño profesional. En este sentido, se genera un potencial en virtud del consenso y organización interpretativa sobre los quehaceres, sentidos y fundamentaciones conceptuales que los/as profesores/as desarrollan en función de una acción didáctica mas pertinente y congruente, tanto a nivel de las orientaciones curriculares nacionales, como en los proyectos educativos locales a los cuales se agencian.

En términos de levantamiento de las categorias, se puede apreciar que las convergencias de las propuestas teóricas (consideradas en la fase de revisión bibliográfica) y el discurso de los docentes en ejercicio, da cuenta de una constante discusión entre lo teórico y lo práctico del desempeño del profesional de educación física. En este sentido, las dimensiones levantadas (Gestión, Acción y Saber Didáctico) permiten definir características que se expresan directamente en el ejercicio profesional, y que, desde la densidad y forma en que se relacionan, permiten observar y evaluar multidimensionalmente el desempeño de los docentes. Lo fundamental dentro de la configuración de las dimensiones mencionadas, con sus respectivos indicadores, se focaliza o adquiere sentido en las propiedades de relación entre ellas. 
Esto último permite interpretar que el proceso de enseñanzaaprendizaje se debe situar especificamente dentro del contexto escolar, es decir, la relacionalidad de las dimensiones se produce en el ejercicio profesional en contextos situados y reales, los cuales, en la medida que son observados e informados, tanto desde la propia experiencia resultante del docente, como de un par observador, puede ir profundizando en niveles mas complejos y superiores de desempeño.

Por otra parte, las categorias develan un énfasis en aspectos que tradicionalemente no son considerados como sustantivos, o mas propios de la disciplina, y que se remiten hacia habilidades $\mathrm{y}$ competencias vinculadas con posicionamientos comunicativos, relacionales y de agenciamiento personal y social. Aspecto que revela el énfasis educativo y cultural del ejercicio profesional, por sobre lo técnico-instrumental.

Finalmente, desde el punto de vista del diseño investigativo, la complementariedad metodológica de las tres fases de investigación (teórica, ingenua y naturalista), permitió configurar un dispositivo preliminar de observación orientado a generar círculos creativos de diálogos en torno a la convergencia de saberes y acciones profesionales. Con ello, se espera aportar con un recurso de desarrollo cualitativo para la Educación Física, potenciando el despliegue de esquemas de observación con grados de mayor complejidad y, simultáneamente, espacios relacionales de trabajo cooperativo orientados a la consistencia, congruencia y pertinencia de las acciones profesionales en este contexto. 

Development of a device for observation of
Physical Education classes at elementary
school level: a naturalist perspective from
teaching experience
Abstract: The present investigation takes place in a
qualitative project sponsored by the Chilean Education
Ministry, in order to develop an observation instrument
in P.E classes mainly in primary public schools. The
previously mentioned project enables dialogue
processes to improve professionals' didactic work in
this area. The qualitative project was developed in
complementary stages: specific literature review,
perceptions of senior PE students and perceptions of
PE teachers who are working at public schools. In
relation to the sources, three didactic processes were
established: management, action and didactic
knowledge. As a result, it was possible to define the
indicators for each dimension, as well as investigate
implementation indicators from the specific instrument.
This report deals mainly with the results and process
associated to the naturalist stage.
Key words: Education, Primary and Secondary.
Physical Education. Observation. Qualitative analysis. 


\begin{tabular}{l} 
Desenvolvimento de um dispositivo para a \\
observaçao de aulas de Educacao Fisica para a \\
educacao básica: uma perspetiva naturalista da \\
experiencia docente \\
Resumo: O presente trabalho sistematiza a \\
abordagem qualitativa de um projeto patrocinado pelo \\
Ministério de Educação do Chile, em função de \\
desenvolver um instrumento de observação de aulas \\
de Educação Física para o Ensino Geral Básico \\
(Educação Básica) nos estabelecimentos com \\
financiamento público. O mencionado instrumento tem \\
como objetivo favorecer processos de diálogo \\
disciplinar e melhora no desempenho didático dos \\
profissionais da área. O trabalho qualitativo se \\
desenvolveu em etapas sucessivas e \\
complementares: revisão de literatura específica, bem \\
como as expressões e percepções de estudantes do \\
último ano do curso de Educação Física e de \\
professores que trabalham em escolas que são \\
subsidiadas com fundos públicos do citado Ministério. \\
Da confluência das mencionadas fontes se \\
estabeleceram três dimensões do desempenho \\
didático, a saber: gestão, ação e saber didático. Como \\
resultados se buscou depurar e definir os indicadores \\
de cada dimensão, como também pesquisar indicações \\
de implementação do instrumento em questão. \\
Palavras-chave: Ensino fundamental e médio. \\
Educação Física. Observaçao. Análise qualitativa. \\
\hline
\end{tabular}

\section{REFERENCIAS}

AHUMADA, Pedro. Hacia una evaluación auténtica del aprendizaje. Barcelona: Paidos Educador, 2005.

ARNOLD, Marcelo. Recursos para la investigación sistémico/constructivista. In: OSORIO, Francisco (Ed.). Ensayos sobre socioautopoiesis y epistemología constructivista. Santiago de Chile. Facultad de Ciencias Sociales Universidad de Chile. 2004.

BLAZQUEZ, Domingo. La evaluación de la educación física. Barcelona: Inde. 2000. 
CASTAÑER, Olaguer; CAMERINO, Olga. La Educación Física en la enseñanza primaria. Barcelona: Inde. 1996.

ESPINA, Mayra. Complejidad, transdisciplina y metodología de la investigación social. Utopía y Praxis Latinoamericana, Maracaibo v.12, n.38, p.29-43. 2007

FREEMAN, Walter. Dinámicas no lineales e intencionalidad: El rol de las teorías cerebrales en las ciencias de la mente. In: IBAÑEZ, Agustin; COSMELLI, Diego. Nuevos enfoques de la cognición. Santiago de Chile: Universidad Diego Portales. 2007. p 27-52.

GLASER, Berney; STRAUSS, Anselm. The discovery of grounded theory: Strategies for qualitative research. Chicago: Aldine. 1967.

HUBRIG, Christa; HERRMANN, Peter. Lösungen in die Schule. Systematisches denken in Unterrich, Beratung und Schulentwicklung. Heildelberg: CarlAuer Verlag. 2010.

IBAÑEZ, Agustin; COSMELLI, Diego. Nuevos enfoques de la cognición. Redescubriendo la dinámica de la acción, la intención y la intersubjetividad. Santiago de Chile: Universidad Diego Portales. 2007.

LÓPEZ Pastor, Victor. Prácticas de evaluación en educación física. Estudios de casos en primaria, secundaria y formación de profesorado. Valladolid: Universidad de Vallalodid. 1999.

NÖE, Alva. Fuera de la cabeza. Por qué no somos el cerebro y otras lecciones de la biología de la consciencia. Barcelona: Kairós. 2010.

PASTOR, Jose. L. Motricidad, ámbitos y técnicas de intervención. Alcalá: UAH. 2007.

RAMOS, Luis; DEL VILLAR, Fernando. La enseñanza de la educación física. Madrid: Editorial Sintesis. 2005.

THOMPSON, Evan. Mind in life. Biology, phenomenology, and the sciences of mind. Cambridge: Belknap Harvard. 2007.

TOPSCH, Wagner. Grundwissen für Schulprakticum und Unterrich. Weinheim: Belz Pädagogik. 2005.

VARELA, Francisco. El fenómeno de la vida. Santiago: Dolmen Ensayo. 2000. 
Apoio financeiro: Ministerio de Educación de Chile, Universidad Austral de Chile.

Nota: os instrumentos e protocolos utilizados na pesquisa poderão ser solicitados diretamente ao autor pelo e-mail: seatoro@gmail.com

Endereço para correspondência:

Sergio Toro Arévalo

Instituto de Filosofía y Estudios Educacionales

Campus Isla Teja $\mathrm{s} / \mathrm{n}$.

Universidad Austral de Chile

Valdivia, Chile

Recebido em: 29-06-2012

Aprovado em: 01-12-2012

Movimento, Porto Alegre, v. 19, n. 01, p. 161-181, jan/mar de 2013. 